\title{
Beteiligungsorientierte Organisation und Innovation
}

\author{
Eva Kirner, Ute Weißfloch, Angela Jäger
}

Unternehmen sind einem ständigen Zwang zur Verbesserung ihres Angebots ausgesetzt. Wer im Wettbewerb bestehen will, muss innovativ sein. Auf der Suche nach neuen Ideen und bei ihrer Umsetzung in Produkte und Prozesse spielen das Wissen und die Erfahrung der Belegschaften eine bedeutende Rolle. Diese Ressource zu aktivieren, ist jedoch keine einfache Aufgabe. Beteiligungsorientierte Arbeitsorganisation und Managementkonzepte, die die Beschäftigten in den Innovationsprozess einbinden, versprechen Erfolg, aber nur wenige Unternehmen in Deutschland setzen dies auch konsequent um. ${ }^{1}$

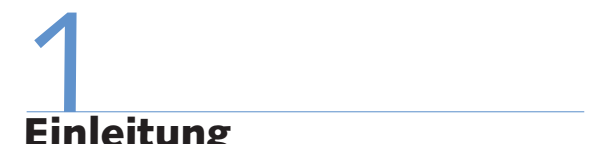

\section{Einleitung}

Einer möglichst breiten Einbeziehung von Beschäftigten in betriebliche Innovationsprozesse wird in der aktuellen Innovationsforschung große Bedeutung für den Innovationserfolg beigemessen. Ihr Wissen und ihre Erfahrung gelten als zentrale Komponenten betrieblicher Innovationsfähigkeit (Tidd/Bessant 2009; Chesbrough 2006). Wir fragen nach dem Zusammenhang zwischen betrieblicher Mitbestimmung und Innovation, d. h. nach Wegen, wie das Erfahrungswissen der Beschäftigten aktiviert und in betriebliche Innovationsprozesse eingebracht werden kann. Im vorliegenden Beitrag untersuchen wir mit statistischen Methoden, wie sich diese Aktivierung im Rahmen der Nutzung beteiligungsorientierter Organisations- und Managementkonzepte sowie einer breiten Verankerung von Innovationskompetenz auf die betriebliche Innovationsfähigkeit auswirkt. $^{2}$

Aus ressourcenorientierter Perspektive (Teece et al. 1997; Barney et al. 2001; Burr 2004) betrachtet, sind die internen Ressourcen und Fähigkeiten eines Unternehmens - und somit endogene Faktoren, nicht vorrangig die Positionierung des Unternehmens am Absatzmarkt - entscheidend für den Markterfolg und die langfristige Wettbewerbsfähigkeit, zu der maßgeblich auch Innovationen beitragen. Das Wissen der Mitarbeiter stellt hierbei einen der wichtigsten Aspekte dar. Unternehmen werden als offene Systeme verstanden, die aufgrund ihrer Elemente und Struktur über bestimmte Fähigkeiten verfügen und diese durch interne Reproduktions- und
Wandlungsprozesse weiterentwickeln und selektieren. Die jeweilige Kombination aus Struktur sowie Ressourcenausstattung ist für jedes Unternehmen einzigartig. Wenn diese unternehmensindividuelle Kombination zielgerichtet und mit Erfolg eingesetzt wird, können daraus schwer imitierbare Wettbewerbsvorteile entstehen. Aus ressourcentheoretischer Sicht sind Unternehmen dann erfolgreich, wenn sie über überlegene oder einzigartige Ressourcen bzw. Ressourcenkombinationen verfügen und diese besser nutzen als ihre Wettbewerber (Barney et al. 1991; Peteraf 1993; Burr 2004; Billerbeck 2003). Sogenannten organisationalen Routinen (Penrose 1959; Freiling 2004) kommt bei der Transformation von Ressourcen in nutzbare Kompetenzen deshalb eine herausragende Rolle zu. Kompetenzen können als die Fähigkeit des Unternehmens verstanden werden, seine Ressourcen erfolgreich einzusetzen und $\mathrm{zu}$ verwenden. Aus dieser Fähigkeit heraus können auch die Kernkompetenzen (Prahalad/Hamel 1990) eines Unternehmens abgeleitet werden. Organisationale Routinen dienen auch der Zusammenführung von verteiltem, individuellem Wissen. An dieser Stelle kommt auf partizipative Arbeitsgestaltung ausgerichteten Organisationskonzepten, die eine Einbeziehung der Beschäftigten bewirken, eine besondere Bedeutung zu (Bessant/Caffyn 1997). Sie können wirkungsvolle Mechanismen sein, das verteilte und insbesondere auch das implizite Wissen der Beschäftigten zusammenzuführen und zugänglich zu machen (Spielkamp/Rammer 2006; Spender 1996). Eine intensive Einbeziehung von Mitarbeitern wird von innovativen Unternehmen auch tatsächlich als einer der wichtigen Erfolgsfaktoren für Innovationen genannt (Kirner et al. 2007).
Verschiedene empirische Studien haben sich bereits mit dem Zusammenhang zwischen der Einbeziehung von Beschäftigten in Form von beteiligungsorientier-

\footnotetext{
Der Beitrag basiert auf Ergebnissen des von der Hans-Böckler-Stiftung geförderten Projekts „Die aktive Rolle der Mitbestimmungsinstitutionen im betrieblichen Innovationsprozess ", das vom Fraunhofer ISI in Karlsruhe und dem Institut für Medienforschung und Urbanistik in Stuttgart gemeinsam bearbeitet wird.

2 Ein zweiter, qualitativer Analyseteil des zugrunde liegenden Projekts beschäftigt sich fallstudienbasiert mit dem Innovationshandeln der Mitbestimmungsinstitutionen (Schwarz-Kocher et al. in diesem Heft). Den analytischen Kern bilden zwei unterschiedliche Wege, implizites Anwender- und Erfahrungswissen der Beschäftigten innovationsförderlich zu aktivieren: einerseits der Einsatz beteiligungsorientierter Organisations- und Managementkonzepte im Betrieb, andererseits können auch die Mitbestimmungsstrukturen Arenen schaffen, in die das Erfahrungswissen eingebracht wird.
}

Eva Kirner, Dr., Betriebswirtin, ist wissen schaftliche Mitarbeiterin am Fraunhofer Institut System- und Innovationsforschung (ISI), Karlsruhe. Arbeitsschwerpunkte: Inno vationsmessung, Innovationsmanagement, Unternehmensnetzwerke. e-mail: eva.kirner@isi.fraunhofer.de Ute Weißfloch, Wirtschaftschemikerin, ist wissenschaftliche Mitarbeiterin am Fraunhofer ISI, Karlsruhe. Arbeitsschwerpunkte: Branchenspezifische Innovationsprozesse, innovative Geschäftsmodelle. e-mail: ute.weissfloch@isi.fraunhofer.de Angela Jäger, Sozialwissenschaftlerin, ist wissenschaftliche Mitarbeiterin am Fraunhofer ISI, Karlsruhe. Arbeitsschwerpunkte: Organisation der Erhebung, Modernisierung der Produktion, quantitative Methoden der Sozial- und Wirtschaftsforschung. e-mail: angela.jaeger@isi.fraunhofer.de 
ten Organisationskonzepten und dem Unternehmenserfolg beschäftigt. Die Ergebnisse existierender Studien sind jedoch nicht einheitlich. ${ }^{3}$ Empirische Studien, die den Zusammenhang zwischen dem Einsatz beteiligungsorientierter Organisationskonzepte und konkreten Innovationsindikatoren messen, liegen zudem bislang kaum vor. ${ }^{4}$ Insbesondere fehlen Erkenntnisse hinsichtlich des Zusammenhangs zwischen dem Einsatz beteiligungsorientierter Organisationskonzepte und nichtmonetären Innovationskenngrößen, die mit Prozessinnovationen verbunden sind. Da Prozessinnovationen sich nicht in jedem Fall direkt auf monetäre Größen wie beispielsweise den Umsatz oder die Kosten auswirken, sind neben der Messung monetärer Innovationskennzahlen auch nichtmonetäre Aspekte wie Qualität, Flexibilität oder Schnelligkeit von Bedeutung. Darüber hinaus wird in bisherigen Arbeiten beim Einsatz beteiligungsorientierter Organisationskonzepte nicht nach der Intensität ihrer innerbetrieblichen Nutzung differenziert. Die Nutzungsintensität bzw. die innerbetriebliche Verbreitung dieser Konzepte beeinflusst jedoch wesentlich die erzielbaren Effekte (Bessant/Caffyn 1997). Auch kommt in bisherigen Studien der Art der betrieblichen Verankerung von Innovationskompetenz als Einflussgröße für Innovation vergleichsweise wenig Beachtung zu, obwohl dieser Aspekt ein wichtiger Anhaltspunkt für die Intensität der Einbeziehung von Beschäftigten in Innovationsprozesse und die erzielbaren Ergebnisse sein kann.

Der vorliegende Beitrag setzt an dieser Stelle an und untersucht anhand von Unternehmensdaten statistische Zusammenhänge zwischen dem Einsatz beteiligungsorientierter Organisations- und Managementkonzepte bzw. der breiten innerbetrieblichen Verankerung von Innovationskompetenz innerhalb von Unternehmen und der betrieblichen Innovationsfähigkeit. Im Rahmen eines ganzheitlichen Innovationsverständnisses unterscheiden wir dabei zwischen vier verschiedenen Innovationsfeldern, die entlang der Produktund Prozessdimension sowie der materiellen und immateriellen Dimension verortet werden können (Abbildung 1). Alle vier Felder können gleichrangig ein mögliches Innovationsziel in Unternehmen sein. Innovationsaktivitäten in einem Feld schließen Aktivitäten in einem anderen Feld nicht aus, im Gegenteil: Es bestehen Wechselwir-

\section{Abb. 1: Vier Felder betrieblicher Innovation}

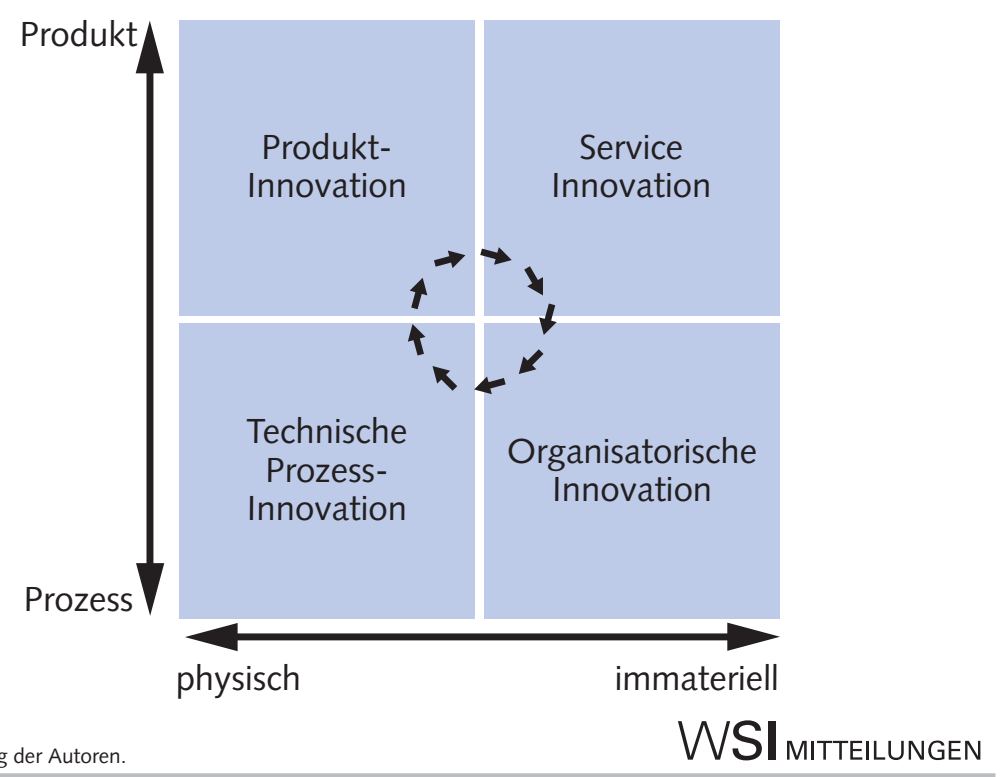

kungen zwischen den verschiedenen Innovationsfeldern. So können beispielsweise Produktinnovationen auch technische oder organisatorische Innovationen nach sich ziehen oder Dienstleistungsinnovationen mit neuen Produkten oder Prozessen zusammenhängen. Unterschiedliche Innovationsarten bringen unterschiedliche Ergebnisse bzw. Innovationserfolge hervor. Während sich die Ergebnisse bzw. Erfolge von Produkt- und Dienstleistungsinnovationen in ihrem Anteil am Umsatz zeigen, sind die Ergebnisse von technischen und organisatorischen Prozessinnovationen weniger eindeutig und teilweise auch nicht direkt monetär messbar. Die Erfolge von Prozessinnovationen können somit am besten durch direkte prozessbezogene Indikatoren gemessen werden.

Die bestmögliche Aktivierung und Nutzung unternehmensindividueller Fähigkeiten und Kompetenzen spielt für Innovationen in allen diesen betrieblichen Innovationsfeldern eine Rolle. Die Aktivierung dieses unternehmensinternen Wissens kann durch die systematische Einbeziehung der Mitarbeiter gefördert werden, da Mitarbeiter die Kompetenzträger in Unternehmen sind. Der vorliegende Beitrag untersucht deshalb anhand von Unternehmensdaten, inwiefern eine stärkere Einbeziehung von Beschäftigten - ausgedrückt durch den Einsatz beteiligungsorientierter Organisations- und Managementkonzepte sowie durch eine breite innerbetriebliche Abstützung von Innovationskompetenz - mit der betrieblichen Innovationsfähigkeit zusammenhängt.

Die folgenden Analysen wurden auf Basis von Daten der Erhebung Modernisierung der Produktion 2006 des Fraunhofer ISI durchgeführt. Diese Daten basieren auf einer Betriebsbefragung und decken das gesamte verarbeitende Gewerbe in Deutschland ab. Sie enthalten Angaben von 1.663 Betrieben. ${ }^{5}$ Inhalt der Befragung sind verfolgte Produktionsstrategien, der Einsatz verschiedener Organisations- und Technikkonzepte, Fragen des Personaleinsatzes und der Qualifikation sowie Leistungsund Innovationsindikatoren.

Wir stellen die Ergebnisse der Analyse in zwei Schritten vor: Zunächst wird die Verbreitung beteiligungsorientierter Organisations- und Managementkonzepte sowie die Art der Verankerung von Innovationskompetenz in deutschen Industrieunternehmen deskriptiv analysiert (Abschnitt 2). Dies soll Aufschluss darüber geben, wie stark diese Arten der Einbeziehung von Beschäftigten in Unternehmen

3 Für einen ausführlichen Überblick über einzelne Studien vgl. Blume/Gerstlberger 2007. Für einen ausführlichen Überblick über empirische Forschungsergebnisse zu Effekten der Gruppenarbeit vgl. Delarue et al. 2008.

4 Eine Ausnahme bildet die Studie von Blume/Gerstlberger (2007).

5 Ausführliche Informationen zur Erhebung "Modernisierung der Produktion“ unter: http://www.isi. fraunhofer.de/i/projekte/erhebung_pi.htm. 
Abb. 2: Verankerung von Innovationswissen in Unternehmen - in \% -

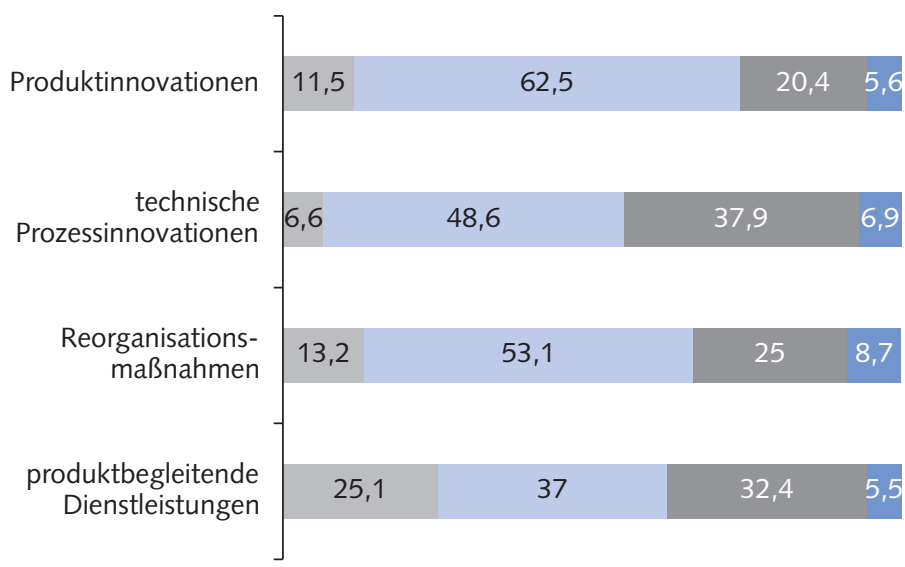

\begin{tabular}{|clc|}
\hline nicht relevant $\begin{array}{l}\text { einzelne oder } \\
\text { wenige Mitarbeiter }\end{array}$ & $\begin{array}{l}\text { Gruppe von } \\
\text { Mitarbeitern }\end{array}$ & Strukturen \\
\end{tabular}

Quelle: Fraunhofer ISI, Erhebung Modernisierung der Produktion 2006.

WSI MITTELLUNGEN

tatsächlich verbreitet sind. Danach wird im Rahmen von Regressionsmodellen (multivariate Analysen) der Zusammenhang zwischen der Einbeziehung von Beschäftigten und der betrieblichen Innovationsfähigkeit untersucht (Abschnitt 3). Innovationsfähigkeit wird hierbei unterteilt in Innovationspotenzial und Innovationserfolg. Das Innovationspotenzial bezieht sich auf innovationsförderliche Rahmenbedingungen, während der Innovationserfolg tatsächlich erreichte Ergebnisse misst. Ein kurzes Fazit rundet den Beitrag ab (Abschnitt 4).

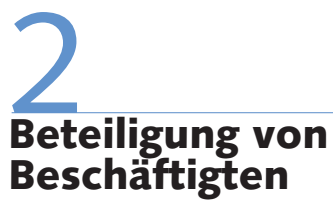

\subsection{BETRIEBLICHE VERANKERUNG VON INNOVATIONSWISSEN}

Die Art der Verankerung von Innovationskompetenz in Unternehmen kann Aufschluss darüber geben, wie stark Mitarbeiter in die Gestaltung und Durchführung von Innovationsprozessen einbezogen werden. Es kann davon ausgegangen werden, dass, je mehr Beschäftigte Innovationskompetenzen besitzen bzw. je fester Innovationskompetenzen im Unternehmen verankert sind, Mitarbeiter umso stärker in Innovationen einbezogen werden. $\mathrm{Zu}$ diesem Aspekt wurde in der Erhebung Modernisie- rung der Produktion 2006 eine gezielte Frage bezüglich der Verankerung von Innovationswissen gestellt. Hierbei wurde bezüglich des Innovationswissens zwischen den Innovationsarten Produktinnovationswissen, Serviceinnovationswissen, technisches Prozessinnovationswissen sowie Reorganisationswissen unterschieden. Die Befragten konnten angeben, ob das jeweilige Innovationswissen im Unternehmen nur bei einzelnen bzw. wenigen Mitarbeitern oder bei einer Vielzahl von Mitarbeitern (einer Gruppe von Mitarbeitern) verankert bzw. ob das jeweilige Innovationswissen im Unternehmen durch Prozesse und Strukturen fest und systematisch verankert ist. Unternehmen konnten zudem angeben, ob die jeweilige Innovationskompetenz für sie überhaupt eine Rolle spielt oder nicht.

Die Ergebnisse zeigen zunächst, dass die Frage der Verankerung von Produktinnovationswissen, Reorganisationswissen und technischem Prozessinnovationswissen für fast alle Unternehmen relevant ist (Abbildung 2). Die gezielte Verankerung von Dienstleistungsinnovationswissen spielt ebenfalls - zwar für etwas weniger, aber immer noch für die überwiegende Mehrheit der Unternehmen - eine Rolle.

Wird die jeweilige Art der Verankerung der verschiedenen Innovationskompetenzen in Unternehmen betrachtet, so zeigt sich hinsichtlich der Verankerung von Produktinnovations- und Reorganisations- wissen ein nahezu identisches Bild: Mehrheitlich (die Hälfte bis zwei Drittel) setzen Unternehmen hierbei auf nur einzelne oder wenige Mitarbeiter. Im Gegensatz da$\mathrm{zu}$ sind bei rund einem Drittel der Betriebe technisches Prozessinnovationswissen sowie Dienstleistungsinnovationswissen durch mehrere Mitarbeiter (Gruppe von Mitarbeitern) abgesichert. Dadurch sind diese Innovationskompetenzen im Durchschnitt breiter im Betrieb verankert. Bemerkenswert einheitlich im Vergleich unterschiedlicher Innovationskompetenzen fällt die Verankerung von Innovationswissen in Form von stabilen Strukturen oder Prozessen aus. Nur ein geringer Anteil der Unternehmen (bis zu knapp einem Zehntel) gibt an, dass für die Verankerung von Innovationskompetenz im Unternehmen Strukturen in Form systematischer Abläufe existieren.

Die Ergebnisse zeigen, dass das für Innovationen relevante Wissen in der Eigenwahrnehmung der Unternehmen vorwiegend auf einzelne Beschäftigte konzentriert ist. Dies könnte damit zusammenhängen, dass Innovationswissen oft nur als Fachoder Expertenwissen wahrgenommen wird. Das Potenzial von unerschlossenem Erfahrungswissen anderer Beschäftigtengruppen wird oftmals noch nicht hinreichend erkannt. Unternehmen verlassen sich häufig ausschließlich auf die Kompetenzen einzelner Mitarbeiter, die das relevante Innovationswissen individuell in ihrer Person vereinen. In diesem Fall existiert weder eine breite Einbeziehung von anderen Beschäftigten noch eine strukturelle Verankerung der Innovationskompetenz, die auch von anderen Beschäftigten genutzt werden könnte.

Immerhin ist - je nach Innovationsart - bei rund einem Fünftel bis $40 \%$ der befragten Unternehmen die Innovationskompetenz personell auf breiterer Basis vorhanden, wenn mehrere Mitarbeiter (eine Gruppe von Mitarbeitern) einbezogen werden.

In der multivariaten Analyse wird es insbesondere von Interesse sein, inwieweit ein Zusammenhang zwischen der breiten Verankerung von Innovationskompetenz und der tatsächlichen Innovationsfähigkeit von Unternehmen statistisch nachweisbar ist. 


\subsection{VERBREITUNG BETEILIGUNGS- ORIENTIERTER MANAGEMENT- KONZEPTE}

Neben der breiten Verankerung von Innovationswissen können beteiligungsorientierte Organisations- und Managementkonzepte ebenfalls eine verstärkte Einbeziehung von Mitarbeitern bei Innovationsprozessen bewirken. Die für diese Analyse ausgewählten Einzelkonzepte weisen partizipative Elemente auf und liefern somit Anhaltspunkte für die direkte Beteiligung der Beschäftigten im jeweiligen Unternehmen. Diese wird anhand des Einsatzes und der Nutzungsintensität der folgenden vier verschiedenen Organisations- und Managementkonzepte untersucht: Gruppenarbeit, Aufgabenintegration (ganzheitliche Aufgabenzuschnitte mit Einbeziehung von planenden, steuernden oder kontrollierenden Funktionen), regelmäßige Personalentwicklungsgespräche und kontinuierliche Verbesserungsprozesse. Diese Konzepte eignen sich potenziell dafür, durch eine stärkere Einbeziehung der Beschäftigten das vorhandene Wissen der Mitarbeiter zu mobilisieren und zu kanalisieren. Im Zusammenhang mit Gruppenarbeit kann zwischen einem weiten und einem eng gefassten Gruppenarbeitskonzept unterschieden werden. Das weite Konzept bezeichnet Gruppenarbeit in allen Ausprägungsformen, unabhängig von spezifischen Merkmalen oder Anforderungen. Unter dem eng gefassten Konzept verstehen wir hingegen Gruppenarbeitsformen, bei denen sich die Mitglieder einer Gruppe gegenseitig vertreten können, d.h. bei denen jedes Gruppenmitglied alle Arbeitsschritte ausführen kann. Dieses eng gefasste Gruppenarbeitskonzept bedeutet eine zusätzliche Konkretisierung des allgemeinen Gruppenarbeitskonzepts (Lay 2008).

Die deskriptive Analyse hinsichtlich der Verbreitung dieser vier Organisations- und Managementkonzepte in der Industrie zeigt (Abbildung 3), dass Gruppenarbeit in der weiten Definition mit über $80 \%$ bereits sehr häufig anzutreffen ist. Allerdings sinkt - wie unsere weiterführenden Analysen (ohne Abbildung) gezeigt haben - der Nutzungsgrad von Gruppenarbeit sehr stark auf $32 \%$, wenn es um ein eng gefasstes Gruppenarbeitskonzept geht, bei dem alle Gruppenmitglieder für alle Aufgaben qualifiziert sind und sich dadurch alle Gruppenmitglieder gegenseitig vertreten können. Bemerkenswert ist zudem, dass nur

\section{Abb. 3: Verbreitung beteiligungsorientierter Organisations- und Managementkonzepte - in \% -}

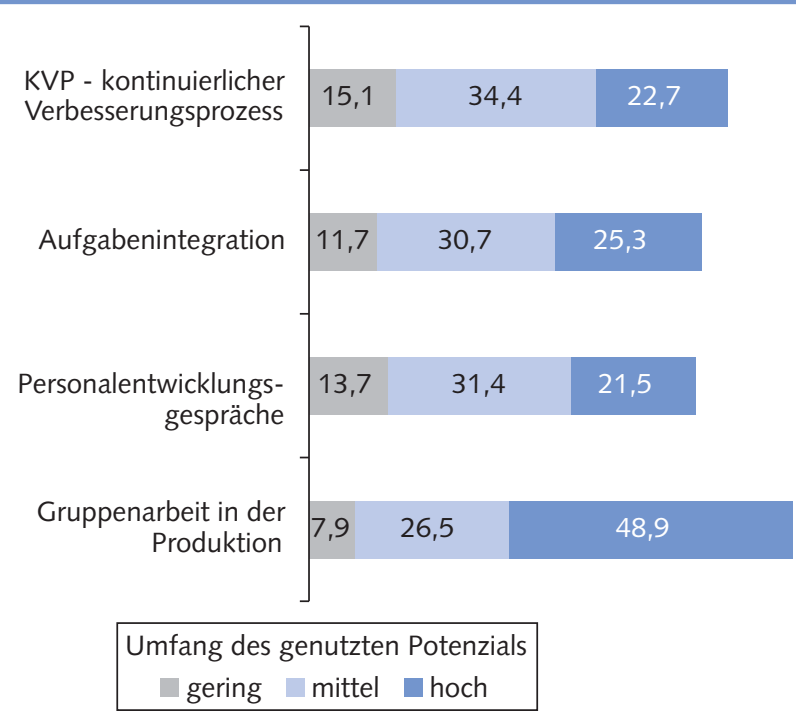

Quelle: Fraunhofer ISI, Erhebung Modernisierung der Produktion 2006.

WSI MITTEILUNGEN

etwas mehr als die Hälfte der Unternehmen, die Gruppenarbeit einsetzen, diese Organisationsform innerhalb des Unternehmens auch intensiv nutzen. Der Anteil der intensiven Nutzer ist bei der engeren Definition von Gruppenarbeit noch deutlich geringer. Nur ein Zehntel der Unternehmen gibt an, dass sie Gruppenarbeit in der engen Definition intensiv nutzen (ohne Abbildung).

Regelmäßige Personalentwicklungsgespräche sind in den untersuchten Betrieben ebenfalls bereits stark verbreitet. Hierbei gibt es wiederum große Unterschiede hinsichtlich der Intensität der Nutzung. Nur rund ein Drittel der Nutzer dieses Konzepts gibt an, dass sie Personalentwicklungsgespräche in hohem Maße im Unternehmen einsetzen. Es ist zu vermuten, dass Personalentwicklungsgespräche meist nur bei bestimmten Mitarbeitergruppen zum Einsatz kommen, aber nicht alle Mitarbeiter betreffen. Die Mehrheit der befragten Unternehmen nutzt Personalentwicklungsgespräche nur teilweise.

Ganzheitliche Aufgabenzuschnitte gemessen anhand des Einsatzes von Aufgabenintegration - werden von zwei Dritteln der befragten Unternehmen praktiziert. Jedoch zeigen sich hier erneut deutliche Unterschiede hinsichtlich der Nutzungsintensität. Ebenso wie regelmäßige Personalentwicklungsgespräche gelten ganzheitliche Aufgabenzuschnitte vermutlich nicht für alle Mitarbeitergruppen, denn lediglich rund ein Drittel der Nutzer dieses Konzepts setzt es innerhalb des Unternehmens auch flächendeckend ein. Kontinuierliche Verbesserungsprozesse (KVP) sind ebenfalls weit verbreitet und werden von über $70 \%$ der befragten Unternehmen genutzt. Trotz dieser hohen Verbreitung des Konzepts gilt bei KVP aber ebenfalls, dass die Nutzung oft auf Teilbereiche des Unternehmens beschränkt bleibt. Lediglich ein Drittel der KVP-Nutzer setzt das Konzept unternehmensweit ein.

Obwohl beteiligungsorientierte Organisations- und Managementkonzepte bereits von der Mehrheit der deutschen Industrieunternehmen genutzt werden, wird deutlich, dass alle Konzepte noch große Potenziale hinsichtlich ihres innerbetrieblichen Verbreitungsgrades aufweisen. Nur eine Minderheit der Unternehmen, die auf diese Konzepte zurückgreifen, setzt sie innerhalb des Unternehmens auch konsequent um. Jedoch kann davon ausgegangen werden, dass der Grad der innerbetrieblichen Nutzung beteiligungsorientierter Konzepte die Stärke ihrer Effekte beeinflusst. Aus diesem Grund wird bei der folgenden multivariaten Analyse des Zusammenhangs zwischen ihrem Einsatz und der betrieblichen Innovationsfähigkeit die jeweilige innerbetriebliche Nutzungsintensität mitberücksichtigt. 


\begin{tabular}{|c|c|c|c|c|}
\hline $\begin{array}{l}\text { Übersicht 1: Wirku } \\
\text { Managementkonze } \\
\text { kompetenz auf das }\end{array}$ & $\begin{array}{l}\text { ng beteiligung } \\
\text { pte und einer } \\
\text { Innovationsp }\end{array}$ & $\begin{array}{l}\text { sorientiert } \\
\text { breiten Ve } \\
\text { otenzial }\end{array}$ & $\begin{array}{l}\text { Organisatio } \\
\text { nkerung von }\end{array}$ & $\begin{array}{l}\text { Is- und } \\
\text { Innovations- }\end{array}$ \\
\hline Innovationspotenzial & $\begin{array}{l}\text { Mitarbeiterquali- } \\
\text { fikation/Anteil } \\
\text { Unqualifizierter }\end{array}$ & $\begin{array}{l}\text { Wettbewerbs- } \\
\text { strategie } \\
\text { Innovation }\end{array}$ & $\begin{array}{c}\text { FuE Kooperation } \\
\text { mit Forschungs- } \\
\text { einrichtungen }\end{array}$ & $\begin{array}{c}\text { FuE Kooperation } \\
\text { mit anderen } \\
\text { Unternehmen }\end{array}$ \\
\hline $\begin{array}{l}\text { Breite Innovationskompe- } \\
\text { tenzverankerung durch } \\
\text { Gruppen von Mitarbeitern } \\
\text { oder Strukturen }\end{array}$ & & & + & \\
\hline $\begin{array}{l}\text { Mitarbeiterorientierung - } \\
\text { Einsatz von KVP, Aufgaben- } \\
\text { integration, Gruppenarbeit } \\
\text { oder von regelmäßigen } \\
\text { Personalentwicklungs- } \\
\text { gesprächen }\end{array}$ & - & & + & + \\
\hline $\begin{array}{l}+ \text { +-: Positiver/negativer Einfluss auf } \\
\text { Signifikanzniveau } \mathrm{p} \leq 0,05 \text {. } \\
\text { Quelle: Zusammenstellung der Auto }\end{array}$ & $\begin{array}{l}f \text { die einzelnen Konzep } \\
\text { orinnen. }\end{array}$ & (1) & & TTEILUI \\
\hline
\end{tabular}

\section{3 \\ Beteiligung von Beschäf- tigten und betriebliche Innovationsfähigkeit}

Bei der Analyse des Zusammenhangs zwischen der Einbeziehung von Beschäftigten und der betrieblichen Innovationsfähigkeit wurden im Rahmen multivariater Analysen multiple lineare und logistische Regressionsmodelle (Long 1997) gerechnet. Um hierbei eventuelle überlagerte Einflüsse von betrieblichen Strukturmerkmalen auszuschließen, wurde in jedem Regressionsmodell auch eine Reihe betrieblicher Strukturmerkmale - Unternehmensgröße, Branchenzugehörigkeit, Art der Produktentwicklung, Produktkomplexität, Seriengröße, Fertigungstiefe, Region (Ost-/Westdeutschland), Exportquote, Qualifikationsstruktur sowie Personalstruktur - kontrolliert. Diese Strukturmerkmale können auch unabhängig von anderen Faktoren einen Einfluss auf die Innovationsfähigkeit von Unternehmen haben und wurden aus diesem Grund in die Analysen einbezogen. Darüber hinaus wurden in den Modellen zum Innovationserfolg weitere Einflussvariablen kontrolliert, die zwar keine strukturellen Ausprägungen im engen Sinne darstellen, aber im Zusammenhang mit der betrieblichen Innovationsfähigkeit stehen. Dazu gehören Kooperationen mit externen Partnern ${ }^{6}$ und die Höhe der Ausgaben für Forschung und Entwicklung (F\&E). ${ }^{7}$

Die zentrale Fragestellung, die den multivariaten Analysen zugrunde liegt, lautet: Welchen Einfluss haben beteiligungsorientierte Organisations- und Managementkonzepte sowie eine breite $\mathrm{Ab}$ - stützung von Innovationswissen in Unternehmen auf die betriebliche Innovationsfähigkeit? Hierbei ist sowohl das Innovationspotenzial eines Unternehmens als auch der tatsächliche Innovationserfolg von Interesse. Als Innovationspotenzial werden Voraussetzungen bzw. Rahmenbedingungen verstanden, die allgemein mit einer starken Innovationsorientierung in Verbindung gebracht werden, während sich der Innovationserfolg auf die tatsächlich erreichten Ergebnisse bezieht.

Hierzu wurden zunächst die vier ausgewählten Konzepte Aufgabenintegration, Personalentwicklungsgespräche, kontinuierlicher Verbesserungsprozess sowie Gruppenarbeit in der engen Definition (Vertretungsmöglichkeit innerhalb der Gruppe) zum Konstrukt „beteiligungsorientierte Organisations- und Managementkonzepte" zusammengefasst. Der Einfluss dieses Konstrukts wurde in insgesamt neun Regressionsmodellen analysiert - vier Modelle zum Innovationspotenzial und fünf Modelle zum Innovationserfolg. Im Rahmen dieser neun Regressionsmodelle wurde zudem die breite Verankerung von Innovationskompetenz analysiert. Es wurde untersucht, wie sich eine breite personelle oder strukturelle Verankerung von Innovationswissen im Unterschied zur Abstützelne Mitarbeiter auf das betriebliche Innovationspotenzial bzw. auf den betrieblichen Innovationserfolg auswirkt.

Im Folgenden werden die Ergebnisse der einzelnen Regressionsmodelle schematisch dargestellt. Im ersten Schritt wurde der Zusammenhang zwischen der breiten Verankerung von Innovationskompetenz sowie dem Einsatz beteiligungsorientierter Organisations- und Managementkonzepte zung des Innovationswissens auf nur ein- und dem Innovationspotenzial näher betrachtet (Übersicht 1). Das Innovationspotenzial eines Unternehmens lässt sich nicht an einem bestimmten Aspekt festmachen, vielmehr handelt es sich hierbei um die Existenz innovationsförderlicher Voraussetzungen, die das Entstehen von Innovationen begünstigen können. Solche Voraussetzungen können beispielsweise gut qualifizierte Mitarbeiter sein oder auch Kooperationen mit externen Partnern, wodurch externes Wissen verfügbar gemacht wird. Zudem kann die strategische Ausrichtung des gesamten Unternehmens auf Innovation ebenfalls eine innovationsförderliche Wirkung entfalten. Davon ausgehend wurde das betriebliche Innovationspotenzial in unserer Analyse mithilfe der folgenden vier Indikatoren gemessen: $\mathrm{Ni}-$ veau der Mitarbeiterqualifikation (Anteil unqualifizierter/angelernter Mitarbeiter), Existenz von Forschungs- und Entwicklungskooperationen mit Unternehmen oder mit Forschungseinrichtungen und die explizite strategische Ausrichtung des Unternehmens auf Innovation. Diese Indikatoren sind zwar noch keine Garantie für tatsächliche Innovationserfolge, jedoch schaffen sie vermutlich innovationsförderliche Rahmenbedingungen, die tatsächliche Innovationserfolge wahrscheinlicher machen. Gut qualifizierte Mitarbeiter können eine Quelle innovativer Ideen sein, aber auch Treiber neuer Innovationsaktivitäten (Tidd/Bessant 2009; Chesbrough 2006). Forschungs- und Entwicklungskooperationen mit anderen Unternehmen oder mit Forschungseinrichtungen können ebenfalls zur Erhöhung der Innovationsfähigkeit beitragen, da durch den Austausch mit externen Partnern neue Impulse aufgenommen, Know-how transferiert und Kompetenzen gebündelt werden können (Pyka 2009; Tidd/Bessant 2009; Gerybadze 2004). Dies erleichtert oft das Hervorbringen von Innovationen. Eine auf Innovationen ausgerichtete betriebliche Wettbewerbsstrategie kann schließlich dazu beitragen, dass im gesamten Unternehmen eine Innovationskultur etabliert und zielgerichtet verfolgt wird.

6 Im Einzelnen: F\&E-Kooperationen im Modell zu Umsatzanteilen mit Produktinnovationen, Servicekooperation im Modell zu Umsatzanteilen mit Serviceinnovationen und Produktionskooperation in den Modellen zur Arbeitsproduktivität, Termintreue und Ausschussquote.

7 Bei Produkt- sowie bei Serviceinnovationen. 
Im Rahmen von vier getrennten Regressionsmodellen wurden zunächst die Zusammenhänge zwischen der Einbeziehung der Beschäftigten und dem so verstandenen Innovationspotenzial unter Kontrolle betrieblicher Strukturmerkmale analysiert.

Die Ergebnisse zeigen, dass der Einsatz beteiligungsorientierter Organisationskonzepte tatsächlich mit einem höheren durchschnittlichen Qualifikationsniveau der Beschäftigten zusammenhängt. Unternehmen, die beteiligungsorientierte Organisations- und Managementkonzepte verstärkt nutzen, haben signifikant geringere Anteile von un- oder angelernten Beschäftigten als Unternehmen, die diese Konzepte nicht oder nur in geringem Maße einsetzen. Dieser Zusammenhang kann in zwei Richtungen interpretiert werden: Entweder fördert eine beteiligungsorientierte Organisationsund Managementkultur in Unternehmen die Höherqualifizierung der Beschäftigten, oder die Unternehmen, deren Beschäftigte bereits ein höheres Qualifikationsniveau besitzen, führen häufiger solche Konzepte ein, weil für den erfolgreichen Einsatz dieser Konzepte erst ein bestimmtes Qualifikationsniveau erforderlich ist. Die breite Verankerung von Innovationskompetenzals weiteres Merkmal der Einbeziehung von Mitarbeitern - scheint hingegen in keinem eindeutigen Zusammenhang zum allgemeinen Qualifikationsniveau der Beschäftigten zu stehen. Hier sind keine statistisch belastbaren Zusammenhänge festzustellen.

Des Weiteren wird deutlich, dass Unternehmen, die beteiligungsorientierte Organisations- und Managementkonzepte einsetzen, häufiger mit Forschungseinrichtungen und auch mit anderen Unternehmen im Bereich Forschung und Entwicklung (F\&E) kooperieren. Ein positiver $\mathrm{Zu}-$ sammenhang besteht darüber hinaus auch zwischen der breiten Verankerung von Innovationskompetenz innerhalb des Unternehmens und F\&E-Kooperation mit Forschungseinrichtungen. Dies lässt vermuten, dass die Offenheit gegenüber externen Innovationspartnern und die Bereitschaft, Wissen auszutauschen, mit steigender Einbeziehung der Beschäftigten generell zunehmen. Je stärker die Beschäftigten durch beteiligungsorientierte Organisations- und Managementkonzepte oder durch eine breite Verankerung der Innovationskompetenz einbezogen werden, um so häufiger werden von diesen Unternehmen Innovationskooperationen mit externen Partnern

\begin{tabular}{|c|c|c|c|c|c|}
\hline \multicolumn{6}{|c|}{$\begin{array}{l}\text { Übersicht 2: Wirkung beteiligungsorientierter Organisations- und } \\
\text { Managementkonzepte und einer breiten Verankerung von Innovations- } \\
\text { kompetenz auf den Innovationserfolg }\end{array}$} \\
\hline Innovationserfolg & $\begin{array}{l}\text { Umsatzanteil } \\
\text { mit neuen } \\
\text { Produkten }\end{array}$ & $\begin{array}{l}\text { Umsatzanteil } \\
\text { mit neuen } \\
\text { Dienst- } \\
\text { leistungen }\end{array}$ & $\begin{array}{l}\text { Arbeits- } \\
\text { produk- } \\
\text { tivität }\end{array}$ & $\begin{array}{l}\text { Termin- } \\
\text { treue }\end{array}$ & $\begin{array}{l}\text { Ausschuss- } \\
\text { quote }^{*}\end{array}$ \\
\hline $\begin{array}{l}\text { Breite Innovationskompetenz- } \\
\text { verankerung durch Gruppen } \\
\text { von Mitarbeitern }\end{array}$ & & + & & + & \\
\hline $\begin{array}{l}\text { Breite Innovationskompetenz- } \\
\text { verankerung durch Strukturen }\end{array}$ & & + & & & \\
\hline $\begin{array}{l}\text { Mitarbeiterorientierung - } \\
\text { Einsatz von KVP, Aufgaben- } \\
\text { integration, Gruppenarbeit oder } \\
\text { von regelmäßigen Personal- } \\
\text { entwicklungsgesprächen }\end{array}$ & & + & & + & - \\
\hline \multicolumn{6}{|c|}{$\begin{array}{l}\text { +/-: Positiver/negativer Einfluss auf die einzelnen Konzepte des Innovationspotenzials, Signifikanzniveau } p \leq 0,05 \text {. } \\
\text { * negativer Einfluss bedeutet, die Ausschussquote sinkt. }\end{array}$} \\
\hline
\end{tabular}

eingegangen. Je mehr Personen mit Innovationen zu tun haben, umso größer sind vermutlich auch die potenziellen Anknüpfungspunkte zu externen Partnern.

Lediglich im Hinblick auf die innovationsbezogene Wettbewerbsstrategie lassen sich keine Zusammenhänge zur Einbeziehung von Beschäftigten feststellen. Die Befunde bezüglich der anderen drei Indikatoren zeigen jedoch, dass Betriebe, die beteiligungsorientierte Organisations- und Managementkonzepte intensiv nutzen, tendenziell ein höheres Innovationspotenzial und somit eine höhere Befähigung für Innovation aufweisen, indem sie häufiger im Bereich Forschung und Entwicklung mit externen Partnern kooperieren und auch tendenziell höher qualifizierte Mitarbeiter beschäftigen. Weiterhin kann gezeigt werden, dass eine breite Verankerung von Innovationskompetenz in Betrieben in Form von strukturierten Abläufen oder durch die Einbeziehung einer Vielzahl von Mitarbeitern statistisch signifikant mit einer häufigeren Kooperationsbereitschaft mit Forschungseinrichtungen zusammenhängt.

Über das Innovationspotenzial hinaus ist jedoch auch von Interesse, wie die stärkere Einbeziehung von Beschäftigten mit konkreten Innovationserfolgen zusammenhängt. Innovationserfolg kann hierbei - abhängig von der Innovationsart - sowohl durch monetäre als auch durch nichtmonetäre Indikatoren gemessen werden. Im Fall von Produkt- und Dienstleistungsinnovationen haben sich der Umsatz mit neuen Produkten bzw. der Umsatz mit neuen Dienstleistungen als guter Erfolgsindikator bewährt. Im Fall von Prozessinnovationen ist die Messung des Innova- tionserfolgs jedoch weniger eindeutig, da mehrere, teilweise nicht-monetäre Faktoren eine Rolle spielen. Um möglichst vielen verschiedenen Dimensionen des Prozessinnovationserfolgs gerecht zu werden, werden im Folgenden die Arbeitsproduktivität als Ausdruck des Effizienzaspekts, die Termintreue als Ausdruck des Zeitaspekts sowie die Ausschussquote als Ausdruck des Qualitätsaspekts herangezogen. ${ }^{8}$ Neben betrieblichen Strukturmerkmalen werden - je nach Innovationsart - auch andere Einflussfaktoren zusätzlich in die Modelle mit einbezogen. Zudem wurde - im Unterschied zur Messung des Innovationspotenzials - bei der Verankerung von Innovationskompetenz zwischen unterschiedlichen Arten von Innovationskompetenzen unterschieden, da eine Differenzierung für die verschiedenen Innovationsergebnisse von Bedeutung ist. Für Produktinnovationen spielt das Wissen über neue Produkte eine Rolle, während für die Termintreue eher das Wissen über organisatorische und technische Prozesse von Bedeutung ist.

Eine breite - entweder personelle oder strukturelle - Verankerung von Innovationswissen in Unternehmen im Vergleich zur Abstützung auf nur einzelne Personen hängt signifikant positiv mit dem Dienstleistungsinnovationserfolg zusammen. Es ist davon auszugehen, dass erfolgreiche Dienstleistungsinnovatoren über ein breites Spektrum an Möglichkeiten verfügen, Kundenanregungen und Kundenwünsche

\footnotetext{
8 Arbeitsproduktivität wird als Umsatz minus Vorleistungen pro Beschäftigten, Qualität durch die Ausschussquote und Termintreue durch den Anteil termingerecht ausgelieferter Aufträge gemessen.
} 
aufzunehmen, auszuwerten und in verbesserte Dienstleistungen umzusetzen. Dies kann entweder durch eine ausreichend hohe Anzahl von Personen geschehen, die im direkten Kundenkontakt stehen und dadurch die Anforderungen erfolgreich identifizieren und umsetzen können, oder durch die Etablierung von strukturierten Abläufen, die Kundenfeedback systematisch aufnehmen und auswerten. Zudem stärkt eine breite personelle Abstützung des Prozessinnovationswissens die Fähigkeit des Unternehmens, Prozesse zuverlässig und stabil zu gestalten, sodass eine hohe Termintreue erreicht werden kann.

Ein signifikant positiver Zusammenhang kann zudem zwischen dem Einsatz beteiligungsorientierter Organisations- und Managementkonzepte und dem Dienstleistungsinnovationserfolg, der Termintreue sowie der Qualität aufgezeigt werden. Unternehmen, die die ausgewählten beteiligungsorientierten Organisations- und Managementkonzepte verstärkt nutzen, weisen im Vergleich zu anderen Unternehmen signifikant höhere Umsatzanteile mit neuen Dienstleistungen, eine signifikant bessere Termintreue und signifikant geringere Ausschussquoten auf als andere Unternehmen. Dies gilt unter Kontrolle aller anderen betrachteten Einflussfaktoren. Der Einsatz der beteiligungsorientierten Organisations- und Managementkonzepte kann die Kompetenzentwicklung im weitesten Sinne und die intensivere Einbeziehung der Mitarbeiter in die Unternehmensabläufe fördern, wodurch ein besseres Verständnis für die Kundenseite und für neue, unterstützende Dienstleistungen geschaffen werden kann. Auch steht die erreichte Qualität in einem direkten Zusammenhang zur breiten Einbeziehung von Mitarbeitern. Wie die vorliegenden Ergebnisse zeigen, kann die Qualität verbessert wer- den, wenn Beschäftigte mehr Verantwortung übertragen bekommen und größere Handlungsspielräume besitzen.

Keine belastbaren Zusammenhänge zeigen sich allerdings hinsichtlich des Umsatzanteils neuer Produkte sowie der Arbeitsproduktivität. Wie die detaillierten Ergebnisse dieser Regressionsmodelle verdeutlichen, wird der Umsatzanteil mit Produktinnovationen zwar durch bekannte Faktoren, wie beispielsweise Ausgaben für Forschung und Entwicklung oder Personalanteil in Forschung und Entwicklung, beeinflusst, ein Zusammenhang zur Beteiligung der Beschäftigten lässt sich auf Basis unserer Analysen jedoch nicht feststellen. Die ausgewählten Organisations- und Managementkonzepte sind Konzepte, die in der Praxis häufig vorrangig im Kontext von Prozessverbesserungen genutzt werden und weniger direkt auf den Produktentwicklungsprozess ausgerichtet sind. Obwohl durch beteiligungsorientierte Konzepte Mitarbeiter stärker auch in Produktentwicklungsprozesse einbezogen werden können, wird diese Möglichkeit im Unternehmensalltag auffällig weniger genutzt. Bei der Arbeitsproduktivität handelt es sich zudem um eine stark aggregierte Kenngröße, die von einer Vielzahl verschiedener Variablen beeinflusst wird. Die Erklärungskraft der betrachteten Formen der Einbeziehung von Beschäftigten ist im Vergleich $\mathrm{zu}$ anderen Aspekten daher nicht groß genug, um belastbare Zusammenhänge aufzuzeigen.

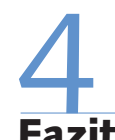

Fazit

Zusammenfassend lässt sich auf Basis der vorgestellten Analysen feststellen, dass so- wohl die breite innerbetriebliche Verankerung von Innovationskompetenzen als auch die intensive Nutzung von beteiligungsorientierten Organisations- und Managementkonzepten in deutschen Industrieunternehmen vergleichsweise wenig verbreitet ist. Meist besitzen nur einzelne Mitarbeiter im Unternehmen innovationsrelevantes Wissen. Oft nutzen Unternehmen beteiligungsorientierte Organisationsund Managementkonzepte zwar in ausgewählten Unternehmensbereichen, aber nicht umfassend. Allerdings kann auf Basis multivariater Untersuchungen gezeigt werden, dass diejenigen Unternehmen, die mehr Mitarbeiter in Innovationen einbeziehen bzw. beteiligungsorientierte Konzepte intensiv nutzen, tendenziell auch eine höhere Innovationsfähigkeit aufweisen. Solche Unternehmen zeigen ein größeres Innovationspotenzial durch höher qualifizierte Mitarbeiter sowie mehr Forschungsund Entwicklungskooperationen, und sie erzielen auch zum Teil bessere Innovationsergebnisse in Form höherer Umsätze mit neuen Dienstleistungen bzw. besserer Qualität und Termintreue. Somit kann für die untersuchten Bereiche ein Zusammenhang zwischen der stärkeren Einbeziehung von Beschäftigten und der betrieblichen Innovationsfähigkeit aufgezeigt werden. Diese Ergebnisse untermauern die in der Innovationsforschung vielfach diskutierte Bedeutung der Beschäftigten für die betriebliche Innovationsfähigkeit. Durch ihre Einbeziehung und Beteiligung können wichtige Innovationsimpulse gesetzt und betriebliche Innovationsaktivitäten auf eine breitere Basis gestellt werden. 


\section{LITERATUR}

Barney, J. B. (1991): Firm resources and sustained competitive advantage, in: Journal of Management 1, S. 99-120

Barney, J. B./Wright, M./Ketchen, D. J. (2001): The resource-based view of the firm: Ten years after 1991, in: Journal of Management 6, S. 625641

Bessant, J./Caffyn, S. (1997): High-involvement innovation through continuous improvement, in: International Journal of Technology Management 1, S. 7-28

Blume, L./Gerstlberger, W. (2007): Determinanten betrieblicher Innovation: Partizipation von Beschäftigten als vernachlässigter Einflussfaktor, in: Industrielle Beziehungen 3, S. 223-244

Billerbeck, H. (2003): Der Zeitfaktor im Innovationsmanagement, Göttingen

Burr, W. (2004): Innovationen in Organisationen, Stuttgart

Chesbrough, H. (2006): Open Innovation. The new imperative for creating and profiting from technology, Harvard

Delarue, A./van Hootegem, G./Procter, S./Burridge, M. (2008): Teamworking and oprganizational performance. A review of survey-based research, in: International Journal of Management Reviews 2, S. 127-148 Freiling, J. (2004): A competence-based of theory of the firm, in: Croucher, R./Kabst, R./Kellerman, R./Matiaske, W. (Hrsg.): Management revue. The international review of management studies. Special issue: Beyond resource based View 1, S. 27-52

Gerybadze, A. (2004): Technologie- und Innovationsmanagement, München
Kirner, E./Maloca, S./Som, O./Rogowski, T./Slama, A. (2007): Kritische Erfolgsfaktoren zur Steigerung der Innovationsfähigkeit. Empirische Studie bei produzierenden KMU, Stuttgart

Lay, G. (2008): Gruppenarbeit in Deutschland - Instrument zur Requalifizierung der Industriearbeit oder leere Worthülse?, in: Arbeit - Zeitschrift für Arbeitsforschung, Arbeitsgestaltung und Arbeitspolitik 1, S. 5-20

Long, J. S. (1997): Regression Models for Categorical and Limited Dependent Variables. Advanced Quantitative Techniques in the Social Sciences, London

Penrose, E. (1959): The theory of the growth of the firm, New York Peteraf, M. A. (1993): The cornerstones of competitive advantage. A resource-based view, Strategic Management Journal 3, S. 179-191 Prahalad, C. K./Hamel, G. (1990): The Core Competence of the Corporation, in: Harvard Business Review 3, S. 79-91

Pyka, A. (Hrsg.) (2009): Innovation networks, Berlin

Spender, J. C. (1996): Making knowledge the basis of a dynamic theory of the firm, in: Strategic Management Journal 2, S. 45-62

Spielkamp, A./Rammer, C. (2006): Balanceakt Innovation. Erfolgsfaktoren im Innovationsmanagement kleiner und mittlerer Unternehmen, Zentrum für Europäische Wirtschaftsforschung (ZEW), Dokumentation 06-04, Mannheim

Teece, D. J./Pisano, G./Shuen, A. (1997): Dynamic capabilities and strategic fit, in: Strategic Management Journal 7, S. 510-533

Tidd, J./Bessant, J. (2009): Managing innovation. Integrating technological, market and organizational change, Chichester 